\title{
Nova Metodologia para Identificação de Componentes em Tintas Comerciais
}

\author{
Rita C. L. Dutra, Marta F. K. Takahashi, Milton F. Diniz \\ Divisão de Química, IAE, CTA
}

Resumo: Uma nova metodologia, incluindo técnicas FTIR, foi desenvolvida e aplicada na separação e caracterização dos principais componentes de tintas alquídicas. Este trabalho demonstra a importância do acoplamento de métodos na análise destes materiais. O método desenvolvido em nossos laboratórios pode ser aplicado, com algumas adaptações específicas e apropriadas, na caracterização de tintas comerciais de composições similares.

Palavras-chave: Tintas, poliálcool, poliácido, hidrólise, FTIR

\section{New Methodology to Identify the Components in Commercial Paints}

Abstract: A new methodology was developed, including FTIR techniques, for separation and characterization of the main ingredients of alkyd paints. This work shows the importance of coupled methods in the analysis of these materials. The method developed in our laboratories can be applied with some specific adaptations in the characterization of commercial paints of similar composition.

Keywords: Paints, polyalcohol, polyacid, hydrolysis, FT-IR.

\section{Introdução}

As principais composições de revestimento são as tintas. As resinas alquídicas modificadas ou não são os componentes-base mais empregados na fabricação de revestimentos ${ }^{[1,2]}$. Encontram aplicações como esmaltes arquitetônicos, tintas para casas (interior e exterior), revestimentos de secagem ao ar e de secagem em estufa e esmaltes para maquinaria ${ }^{[3]}$.

Resinas alquídicas (poliéster) podem ser produzidas a partir de vários tipos de poliálcool, ácido, poliácido e ácido graxo monobásico, disponíveis em uma numerosa distribuição de massas moleculares ${ }^{[1]}$.

Entre os poliálcoois empregados para a preparação de resinas alquídicas destaca-se o glicerol, seguido de pentaeritritol. Misturas de pentaeritritol e glicol etilênico são extensivamente usadas na preparação de resinas alquídicas de cadeias médias e curtas contendo $30-50 \%$ de ácidos graxos ${ }^{[1,2]}$. O comprimento em óleo indica o teor utilizado deste componente. A resina curta contém até $45 \%$ de óleo, a média, de 45 a $55 \%$ e a longa , acima de $55 \%$.

O poliácido mais importante para preparação de resinas alquídicas é o ácido ftálico, produzido e usado na forma de seu anidrido. Os glicerídeos e seus ácidos graxos constituem um grupo de matérias-primas importantes pois afetam diretamente a secagem, flexibilidade, retenção de cor e brilho, solubilidade e custo ${ }^{[1]}$.

Com a exigência do mercado por tintas com menos cheiro e cores mais duráveis, as empresas estão investindo em novas tecnologias. Estes investimentos envolvem desde a adoção de máquinas tintométricas, já bastante comuns em lojas especializadas em tintas, ao aprimoramento das preparações básicas de tintas e dos aditivos, por exemplo, os pigmentos, englobando ainda a adoção de novos solventes, como butanol, butanodiol, acetato de butila e hexanodiol e o aperfeiçoamento de sistemas de cura por radiação $\mathrm{UV}^{[4]}$.

Paralelamente a estas mudanças, há a necessidade de aprimoramento dos métodos de análise de tintas existentes e o desenvolvimento de novos métodos.

Autor para correspondência: Rita C. L. Dutra, Divisão de Química, IAE/CTA, Praça Marechal do Ar Eduardo Gomes, 50, Vila das Acácias, CEP: 12228-904, São José dos Campos, SP. E-mail: ritad@iae.cta.br 
As técnicas de análise instrumental, apesar de requererem equipamentos e mão de obra especializados, são importantes dentro da indústria de tintas pois fornecem informações úteis para a pesquisa de novas formulações bem como para o controle de qualidade da produção das formulações já estabelecidas.

Entre as técnicas instrumentais aplicáveis à análise de tintas e/ou seus constituintes destaca-se a espectroscopia no infravermelho (dispersiva IR e com transformada de Fourier, FTIR) ${ }^{[1,5]}$. Aplicações para a espectroscopia FTIR na indústria de tintas e de revestimentos existem em quase todas as etapas do desenvolvimento do produto e manufatura. Como um método rápido e sensível para estabelecer diferenças sutis nas estruturas de materiais orgânicos, a análise FTIR é valiosa para o controle de qualidade de matérias primas e formulações; para o exame de revestimentos, sejam líquidos ou secos; para identificar componentes; para analisar efeitos de processos como secagem ou exposição às intempéries; e para pesquisa, como a única técnica capaz de fornecer, a baixo custo, informações detalhadas de um produto novo ou competidor.

Basicamente todos os compostos orgânicos e inorgânicos usados na indústria de tintas podem ser analisados por FTIR, por exemplo, as resinas, pigmentos, cargas, solventes e aditivos. Resinas alquídicas e outros polímeros usados em tintas têm sido exaustivamente analisados por FTIR, inclusive envolvendo estudos de decomposição ${ }^{[6-9]}$. Esta técnica é amplamente usada para a identificação de aglutinantes, pigmentos e cargas em tintas nas investigações da ciência forense ${ }^{[10]}$.

A revisão bibliográfica mostrou que muitas resinas alquídicas podem ser produzidas a partir de uma variedade de materiais de partida, que afetam as propriedades finais das tintas e são usados para atender as exigências do mercado. Mostrou também que, paralelamente às mudanças de formulações de tintas, existe a necessidade de aprimoramento e desenvolvimento dos métodos de análise. Esta revisão ressaltou ainda que a análise FTIR, por ser uma técnica de identificação, destaca-se entre as metodologias usadas por requerer menor tempo de análise e não necessitar de derivatizações dos materiais, com reagentes específicos, para suas caracterizações.

Assim, a partir de amostras comercias de tintas à base de resinas alquídicas iniciou-se o desenvolvimento de novas metodologias, incluindo a aplicação de hidrólise e análise FTIR dos materiais de partida, glicóis e ácidos, componentes destas resinas. Este enfoque foi adotado por não ter sido encontrada, no levantamento bibliográfico efetuado, citação de aplicação deste tipo de metodologia na análise de tintas. Portanto, o objetivo desta pesquisa consiste em adaptar e adequar a metodologia convencional e desenvolver nova metodologia para a caracterização por FTIR de tintas comercias baseadas em resinas alquídicas.

\section{Experimental}

\section{Materiais}

Tinta alquídica, de cor branca, contendo resina longa, ou seja, acima de 55\% em óleo, (soja, no caso da amostra) e pentaeritritol, que será denominada de TALP para facilitar a citação (amostra de referência); pentaeritritol MERCK (amostra de referência); esmalte alquídico preto fosco, que será denominado de EPFA (amostra desconhecida).

\section{Condições de Execução}

A análise espectroscópica FTIR foi realizada utilizando o espectrômetro FTIR PERKIN-ELMER 1750 (condições: região de 4000 a $500 \mathrm{~cm}^{-1}$, resolução $4 \mathrm{~cm}^{-1}$, ganho 1,40 varreduras). Para obtenção de alguns espectros, foram aplicados comandos, normalmente permitidos para a melhora da apresentação das absorções, tais como: alinhamento de linha de base (FLAT), expansão de bandas (ABEX) e suavização de ruídos (SMOOTH), utilizando-se fatores adequados e permitidos pelo espectrofotômetro utilizado.

\section{Procedimentos Aplicados}

São apresentados métodos, incluindo técnicas de separação, e preparação das amostras para análise espectroscópica FTIR. Esses métodos incluem o denominado pelos autores de convencional, que abrange a análise da tinta segundo técnicas FTIR conhecidas para preparação de amostras (método 1), e a metodologia que acopla a hidrólise da resina da tinta, após a separação deste polímero-base por tratamento com solventes adequados, e técnicas FTIR para análise dos materiais separados (método 2), que constitui uma das principais contribuições do trabalho.

As técnicas de separação utilizadas nos dois métodos para a análise das amostras de tinta referem-se a tratamentos com solventes adequados, de- 
gradação térmica e condensação de vapores (método 1) e hidrólise (método 2). As técnicas de preparação de amostras para análise espectroscópica FTIR utilizadas para as amostras TALP e EPFA foram: filme líquido capilar, filme vazado, pastilha e pirólise.

\section{Método 1 (Convencional)}

Basicamente envolve a análise direta da amostra de tinta sem tratamento; análise de resinas e cargas, por meio de tratamento com solventes; avaliação de componente ácido da resina por pirólise; e de solventes contidos na amostra, por meio de condensação de vapores. Os passos encontram-se descritos a seguir:

1. Analisar a amostra de tinta tal como recebida segundo a técnica filme líquido capilar;

2. Tratar a amostra de tinta com acetona à quente, próximo ao ponto de ebulição do solvente, centrifugar, separar as fases, evaporar a parte solúvel sobre vidro relógio e analisar o resíduo segundo a técnica filme vazado;

3. Separar a parte insolúvel da amostra, obtida após tratamento com acetona à quente $\mathrm{e}$ centrifugação, e analisar segundo a técnica pastilha. A calcinação da amostra de tinta ou da parte insolúvel em acetona é uma técnica alternativa, que apresenta resultados similares;

4. Evaporar o solvente da amostra até obter um filme. Tratar parte deste filme com éter à quente, pirolisar e analisar o líquido obtido sob forma de pastilha;

5. Colocar, aproximadamente, $10 \mathrm{~mL}$ da tinta em um béquer de $50 \mathrm{~mL}$, tampar o béquer com vidro de relógio e colocar sobre este água gelada e aquecer, aproximadamente a $100{ }^{\circ} \mathrm{C}$ em placa de aquecimento. Os vapores condensarão sob o vidro de relógio, sendo que as primeiras gotas devem ser descartadas e as próximas recolhidas para análise sob a forma de filme líquido.

\section{Método 2}

O método abrange a hidrólise do polímero-base da tinta, para a análise dos componentes ácidos e glicóis contidos nesta resina, e ácidos graxos contidos nas tintas. Os passos são descritos abaixo:

1. Pesar $50 \mathrm{~g}$ da tinta e precipitar o seu polímero - base (resina alquídica) utilizando $250 \mathrm{~mL}$ de éter de petróleo;
2. Centrifugar para separação de cargas;

3. Filtrar e secar;

4. Dissolver $10 \mathrm{~g}$ da resina alquídica, precipitada e seca em acetona (pouca quantidade);

5. Saponificar com $250 \mathrm{ml}$ de uma solução aproximadamente $0,5 \mathrm{~N}$ de $\mathrm{KOH}$ alcoólico por uma (01) hora;

6. Depois que a mistura reacional estiver à temperatura ambiente, filtrar os sais precipitados;

7. Obter os espectros FTIR dos sais, segundo a técnica pastilha;

8. Acidificar o filtrado ligeiramente ( $\mathrm{pH} \mathrm{6-7)} \mathrm{com}$ $\mathrm{HCl}$ concentrado;

9. Filtrar e evaporar o filtrado até $5 \mathrm{ml}$ (concentrar);

10. Transferir a solução concentrada para um funil de separação usando $15 \mathrm{ml}$ de $\mathrm{H}_{2} \mathrm{O}$ (adição aos poucos e agitar);

11. Extrair com éter $(15 \mathrm{ml})$, adicionando aos poucos com agitação. Haverá uma separação em duas fases: aquosa, parte relativa aos glicóis e etérea, relativa aos ácidos graxos;

12. Tornar a camada aquosa ligeiramente alcalina (pH 7-8), com adição de KOH;

13. Secar através de evaporação;

14. Extrair o resíduo evaporado com $15 \mathrm{ml}$ de etanol;

15. Filtrar e secar o filtrado com corrente de ar seco. O resíduo deste tratamento representa a fração alcoólica (glicóis);

16. Obter espectro FTIR, segundo a técnica pastilha;

17. Lavar a fase etérea duas vezes com água destilada;

18. Secar a solução etérea, em temperatura ambiente, usando corrente de ar para evaporar o excesso de solvente e, se necessário, usar dessecador à vácuo;

19. Obter o espectro FTIR, segundo a técnica de filme líquido. No caso de pequena quantidade de amostra, pode-se também optar pela técnica pastilha. Esta etapa corresponde à análise dos ácidos graxos.

\section{Resultados e Discussão}

Este item contém a avaliação dos espectros das amostras analisadas segundo os métodos 1 e 2 . Optou-se por não incluir os espectros dos produtos intermediários da metodologia e de referência dos 
produtos finais, atribuindo-se somente as bandas características, porém é citada a literatura consultada.

Com relação à estrutura química dos componentes das tintas analisadas, basicamente tem-se: as resinas alquídicas, que contém ligações éster do tipo ${ }^{[1]}$ -[CO- $\left(\mathrm{CH}_{\mathrm{n}} \mathrm{COO}-\mathrm{CH}_{\mathrm{n}}-\mathrm{O}\right]-$, dependente do tipo de glicol e ácido utilizado em sua preparação; óleo de soja, um ácido graxo, basicamente do tipo $\left(\mathrm{C}_{18} \mathrm{H}_{22}\right.$ $\mathrm{O}_{2}$ ), e os poliálcoois, que são geralmente compostos bifuncionais que possuem as funções éter (-O-), álcool (-OH) ou éster (-COOH). Os outros componentes, tais como anidrido, solventes aromáticos e cargas contém estruturas bastante divulgadas na literatura ${ }^{[1]}$.

São usados símbolos para indicar os modos vibracionais, a saber: $v$ (estiramento); $v a$ - estiramento assimétrico; $v_{\mathrm{s}}$ - estiramento simétrico; $\delta_{\mathrm{a}}$ - deformação angular assimétrica fora do plano; $\delta_{\mathrm{s}}$ - deformação angular simétrica.

\section{Análise por FTIR da amostra TALP}

Desde que havia a informação que a formulação da tinta possuía resina alquídica, o pentaeritritol como poliálcoool da resina e óleo de soja como ácido graxo, a metodologia teve como meta principal a caracterização destes compostos e, consequentemente, a verificação da aplicabilidade às amostras desconhecidas.

\section{Análise por FTIR da amostra TALP segundo método convencional - método 1 :}

\section{Análise da amostra TALP como recebida}

A Figura 1 a contém as absorções FTIR da amostra TALP analisada tal como recebida, ou seja, sem tratamento. Suas principais absorções ${ }^{[5]}$ estão em: 2924 $\mathrm{cm}^{-1}-\mathrm{v}_{\mathrm{a}}\left(\mathrm{CH}_{2}\right), 2853 \mathrm{~cm}^{-1}-\mathrm{v}_{\mathrm{s}}\left(\mathrm{CH}_{2}\right), 1731 \mathrm{~cm}^{-1}-\mathrm{v}(\mathrm{C}=\mathrm{O}$ em éster $), 1580 \mathrm{~cm}^{-1}-\mathrm{v}(\mathrm{C}=\mathrm{C}$ aromático $), 1459 \mathrm{~cm}^{-1}-\mathrm{v}$ $\left(\mathrm{C}=\mathrm{C}\right.$ aromático), $\delta_{\mathrm{s}}\left(\mathrm{CH}_{2}\right)$ e $\delta_{\mathrm{a}}\left(\mathrm{CH}_{3}\right), 1379 \mathrm{~cm}^{-1}-\delta_{\mathrm{s}}\left(\mathrm{CH}_{3}\right)$, $1269 \mathrm{~cm}^{-1}-\mathrm{v}(\mathrm{COOC})$. As absorções em 1119, $1066 \mathrm{e}$ $948 \mathrm{~cm}^{-1}$, associadas ao seu formato, podem sugerir a presença do grupo $\mathrm{SiO}$, possivelmente da carga existente na tinta. As bandas observadas em 3673 e 3623 $\mathrm{cm}^{-1}-\mathrm{v}(\mathrm{OH})$, podem fazer parte do espectro da carga, o que só será confirmado após tratamento para separação de componentes. Esse conjunto de dados indica a presença de composto orgânico à base de éster e sugere também que haja material inorgânico, provavelmente carga contendo ligações $\mathrm{SiO}$ e $\mathrm{OH}$, tais como às encontradas em silicatos ${ }^{[11 \mathrm{a}]}$ na composição da amostra TALP.

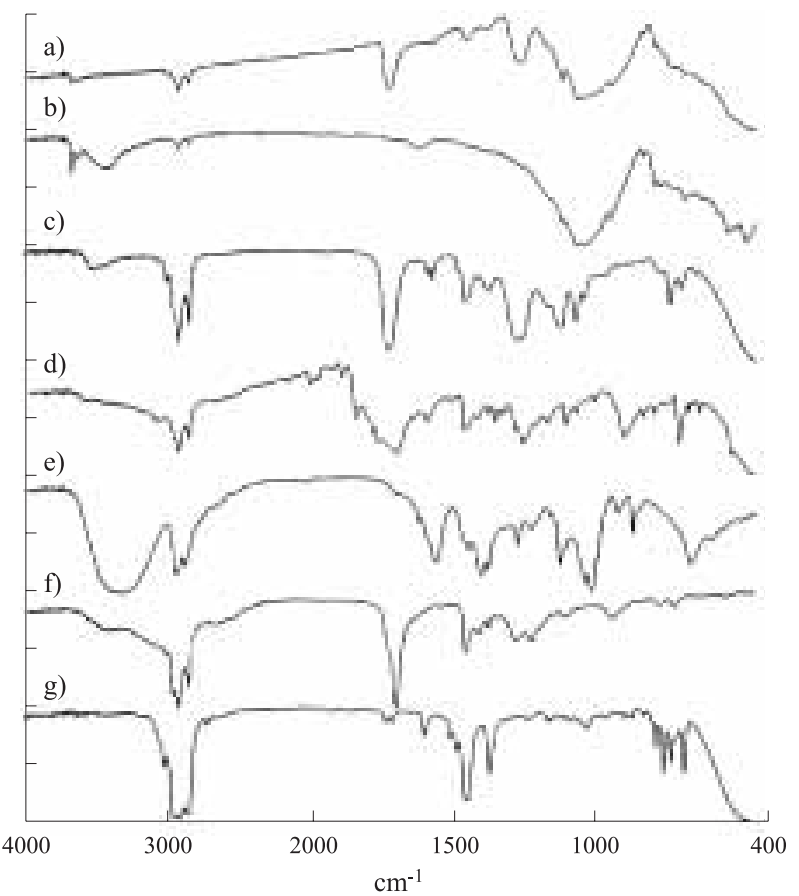

Figura 1. Espectros FTIR da amostra TALP e de seus componentes separados após aplicação da metodologia desenvolvida (métodos 1 e 2)

Análise da amostra TALP após tratamento com solventes

Na tentativa de separar os componentes evidenciados na análise direta do material, a amostra foi tratada com acetona, sendo obtidos os espectros das partes solúvel e insolúvel.

A Figura $1 \mathrm{~b}$ mostra as absorções FTIR do pó (carga) obtido após tratamento da amostra TALP com acetona (parte insolúvel). As principais absorções revelam a presença de silicato, similar à estrutura encontrada em silicato de alumínio ${ }^{[11 a]}$.

É válido lembrar que a análise FTIR não é indicada para avaliação de metais, sendo necessária a utilização de outros métodos, tal como absorção atômica. A indicação da estrutura ser similar à do silicato de alumínio se deve ao formato das absorções. Entretanto, como apoio ao dado espectral, é citada na literatura a utilização de silicato de alumínio como pigmento inerte em tintas ${ }^{[12]}$.

As principais absorções do espectro FTIR do resíduo obtido após tratamento da amostra TALP com acetona (parte solúvel - Figura $1 \mathrm{c}$ ), associadas aos seus grupos funcionais ${ }^{[5]}$, são observadas em: $3533 \mathrm{~cm}^{-1}-\mathrm{v}(\mathrm{OH}), 2925 \mathrm{~cm}^{-1}-\mathrm{v}_{\mathrm{a}}\left(\mathrm{CH}_{2}\right), 2854 \mathrm{~cm}^{-1}$ - $v_{\mathrm{s}}\left(\mathrm{CH}_{2}\right), 1734 \mathrm{~cm}^{-1}-\mathrm{n}(\mathrm{C}=\mathrm{O}$, em éster $), 1580 \mathrm{~cm}^{-1}$ $v(\mathrm{C}-\mathrm{C}$ aromático $), 1465 \mathrm{~cm}^{-1}-\mathrm{v}(\mathrm{C}=\mathrm{C}$ aromático $)$, $\delta_{\mathrm{s}}\left(\mathrm{CH}_{2}\right)$ e $\delta_{\mathrm{a}}\left(\mathrm{CH}_{3}\right), 1379 \mathrm{~cm}^{-1}-\delta_{\mathrm{s}}\left(\mathrm{CH}_{3}\right), 1273,1122$, 
1072 e $1042 \mathrm{~cm}^{-1}$ - v(COOC), 742 e $706 \mathrm{~cm}^{-1}-\delta(\mathrm{C}-\mathrm{H}$ - substituição aromática).

Esse conjunto de absorções confirma que a resina alquídica é o polímero-base da tinta ${ }^{[13 a]}$.

\section{Análise do pirolisado da amostra TALP}

As principais absorções observadas no espectro FTIR do pirolisado da tinta TALP, após a amostra ter sido tratada com éter (Figura $1 \mathrm{~d}$ ), estão em: $1853 \mathrm{e}$ $1766 \mathrm{~cm}^{-1}-\mathrm{v}(\mathrm{C}=\mathrm{O}$ em anidrido cíclico $) ; 1259,1110$ e $910 \mathrm{~cm}^{-1}$ - v(COOC em anidrido cíclico), e caracterizam a presença de anidrido cíclico de 5 membros ${ }^{[5]}$.

Como anidrido ftálico é um dos componentes ácidos utilizados em resinas alquídicas ${ }^{[1,2]}$, o espectro do pirolisado foi comparado ao do referido composto ${ }^{[11 b]}$ e a similaridade observada confirmou que este anidrido é um dos materiais de partida da resina.

Análise do líquido obtido após condensação de vapores da amostra TALP

A Figura $1 \mathrm{~g}$ revela as absorções FTIR do líquido obtido após condensação de vapores da amostra TALP. Suas principais bandas, associadas aos respectivos grupos funcionais ${ }^{[5]}$, estão em: $3027 \mathrm{~cm}^{-1}-\mathrm{v}(\mathrm{C}$ $\mathrm{H}), 2931 \mathrm{~cm}^{-1}-\mathrm{v}_{\mathrm{a}}\left(\mathrm{CH}_{2}\right), 2872 \mathrm{~cm}^{-1}-\mathrm{v}_{\mathrm{a}}\left(\mathrm{CH}_{3}\right), 1607$, 1516,1495 e $1456 \mathrm{~cm}^{-1}-v(\mathrm{C}=\mathrm{C}$ aromático $), 1456$ $\mathrm{cm}^{-1}-\delta_{\mathrm{a}}\left(\mathrm{CH}_{3}\right)$ e $\delta_{\mathrm{s}}\left(\mathrm{CH}_{2}\right), 1377 \mathrm{~cm}^{-1}-\delta_{\mathrm{s}}\left(\mathrm{CH}_{3}\right), 795$, 768,742 e $697 \mathrm{~cm}^{-1}$ - $\delta(\mathrm{C}-\mathrm{H}$ - substituição aromática).

Essas absorções sugerem a presença de hidrocarboneto aromático ou de suas misturas ${ }^{[11 c]}$.

\section{Análise por FTIR da amostra de Tinta TALP segundo o método 2}

As principais absorções FTIR observadas para o sal obtido após saponificação da resina que compõe a tinta TALP revelam a presença de sal de ácido ${ }^{[5]}(1605$ e $1568 \mathrm{~cm}^{-1}-\mathrm{v}_{\mathrm{a}}(\mathrm{C}=\mathrm{O}), 1405,1381 \mathrm{~cm}^{-1}-\mathrm{v}_{\mathrm{s}}(\mathrm{C}=\mathrm{O}), \mathrm{o}$ que está de acordo com a metodologia (método 2 passo 7).

As principais absorções do espectro FTIR do resíduo obtido após a aplicação do método 2 (passos $10 \mathrm{a} 16)$ à tinta TALP estão em: $3338 \mathrm{~cm}^{-1}-\mathrm{v}(\mathrm{OH})$, $2941 \mathrm{~cm}^{-1}-\mathrm{v}_{\mathrm{a}}\left(\mathrm{CH}_{2}\right), 2885 \mathrm{~cm}^{-1}-\mathrm{v}_{\mathrm{s}}\left(\mathrm{CH}_{2}\right), 1567 \mathrm{~cm}^{-1}$ - $\mathrm{v}_{\mathrm{a}}(\mathrm{C}=\mathrm{O}), 1452 \mathrm{~cm}^{-1}-\delta_{\mathrm{s}}\left(\mathrm{CH}_{2}\right), 1407 \mathrm{~cm}^{-1}-\delta(\mathrm{OH}) \mathrm{e}$ $1018 \mathrm{~cm}^{-1}-\mathrm{v}(\mathrm{C}-\mathrm{O})$.

Este conjunto de absorções, associado ao seu formato, sugere a presença básica de composto alcoólico primário e ainda do sal ácido ${ }^{[5]}$. As principais absorções observadas para o glicol confirmam a presença de pentaeritritrol (Figura 1 e).

As principais bandas observadas no espectro FTIR do resíduo obtido após aplicação do método 2 (pas-

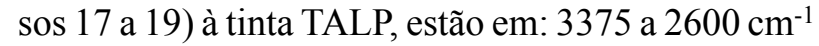
- v (OH), $2927 \mathrm{~cm}^{-1}-\mathrm{v}_{\mathrm{a}}\left(\mathrm{CH}_{2}\right), 2855 \mathrm{~cm}^{-1}-\mathrm{v}_{\mathrm{s}}\left(\mathrm{CH}_{2}\right)$, $1708 \mathrm{~cm}^{-1}-\mathrm{v}(\mathrm{C}=\mathrm{O}), 1461 \mathrm{~cm}^{-1}-\mathrm{v}_{\mathrm{a}}\left(\mathrm{CH}_{3}\right) \mathrm{e} \delta_{\mathrm{s}}\left(\mathrm{CH}_{2}\right)$, $1415 \mathrm{~cm}^{-1}-\delta(\mathrm{OH}), 1381 \mathrm{~cm}^{-1}-\delta_{\mathrm{s}}\left(\mathrm{CH}_{3}\right), 1288 \mathrm{~cm}^{-1}-$ $v(\mathrm{C}-\mathrm{O})$ e $969 \mathrm{~cm}^{-1}-\delta(\mathrm{OH})$. Tais bandas indicam a presença de ácido orgânico ${ }^{[5]}$.

Como é conhecido que a formulação contém óleo de soja, então este espectro (Figura $1 \mathrm{f}$ ) foi comparado ao do referido óleo [13b]. A similaridade observada confirma que este componente faz parte da tinta.

\section{Análise por FTIR da amostra EPFA}

A metodologia foi aplicada à amostra com o objetivo de identificar e caracterizar seus principais componentes.

\section{Análise por FTIR da amostra EPFA segundo método convencional - método 1}

\section{Análise da amostra EPFA tal como recebida}

A Figura 2 a revela as absorções FTIR da amostra EPFA analisada tal como recebida. Suas principais absorções, associadas aos seus respectivos grupos funcionais ${ }^{[5]}$, estão em: $3504 \mathrm{~cm}^{-1}-\mathrm{v}(\mathrm{OH}), 2926 \mathrm{~cm}^{-1}$ $-\mathrm{v}_{\mathrm{a}}\left(\mathrm{CH}_{2}\right), 2855 \mathrm{~cm}^{-1}-\mathrm{v}_{\mathrm{s}}\left(\mathrm{CH}_{2}\right), 1728 \mathrm{~cm}^{-1}-\mathrm{v}(\mathrm{C}=\mathrm{O} \mathrm{em}$ éster), 1269, 1121, 1072 e $1041 \mathrm{~cm}^{-1}-v$ (COOC). As absorções em 1494, 873 e $855 \mathrm{~cm}^{-1}$, associadas ao seu formato, podem sugerir a presença do ânion carbonato, possivelmente da carga existente na tinta, o que só será confirmado após tratamento para separação de componentes. Este conjunto de dados indica a presença de composto orgânico à base de éster e sugere também que haja material inorgânico, provavelmente uma carga contendo ânion carbonato.

\section{Análise da amostra EPFA após tratamento com} solventes

$\mathrm{Na}$ tentativa de separar os componentes evidenciados na análise direta do material, a amostra foi tratada com acetona, sendo obtidos os espectros das partes solúvel e insolúvel. A Figura $2 \mathrm{~b}$ revela as absorções FTIR do resíduo obtido após calcinação do pó, obtido após tratamento da amostra com acetona (parte insolúvel). As principais absorções confirmam a presença de carbonato, similar à estrutura encontrada em carbonato de cálcio ${ }^{[11 \mathrm{~d}]}$. 


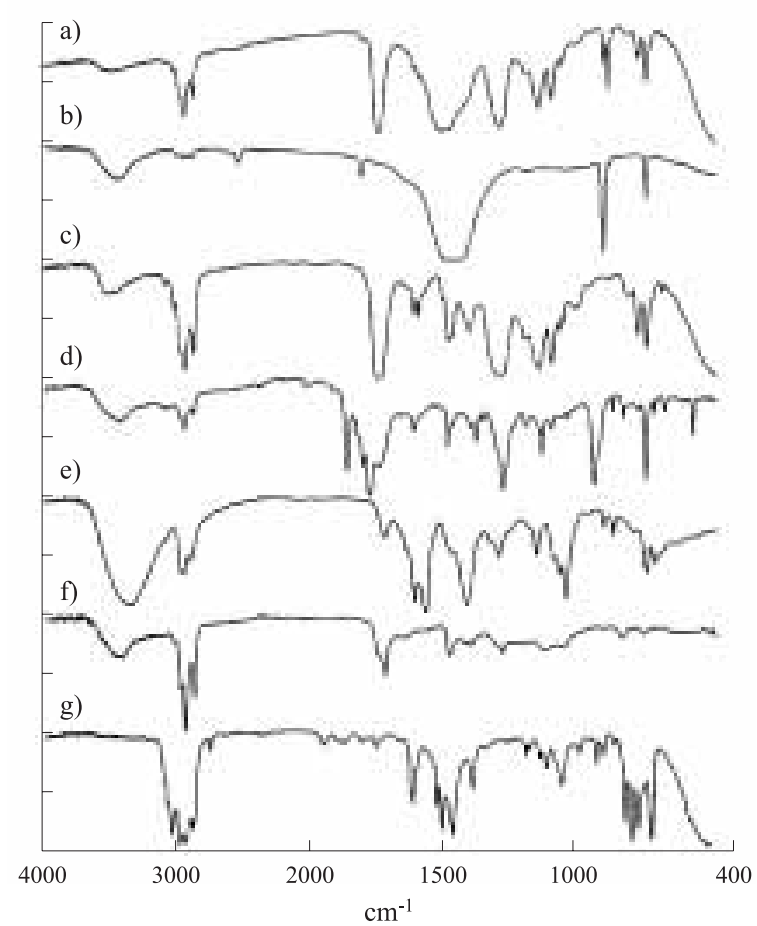

Figura 2. Espectros FTIR da amostra EPFA e de seus componentes separados após aplicação da metodologia desenvolvida (métodos 1 e 2).

A indicação da estrutura ser similar à relativa ao carbonato de cálcio deve-se ao formato das absorções. Entretanto como apoio ao dado espectral, é citada na literatura ${ }^{[12]}$ a utilização de carbonato de cálcio como pigmento inerte em tintas.

As principais absorções FTIR do resíduo obtido após tratamento da amostra EPFA com acetona (parte solúvel - Figura $2 \mathrm{c}$ ) são observadas em: $3516 \mathrm{~cm}^{-1}$ $-\mathrm{v}(\mathrm{OH}), 2927 \mathrm{~cm}^{-1}-\mathrm{v}_{\mathrm{a}}\left(\mathrm{CH}_{2}\right), 2855 \mathrm{~cm}^{-1}-\mathrm{v}_{\mathrm{s}}\left(\mathrm{CH}_{2}\right)$, $1728 \mathrm{~cm}^{-1}-v(\mathrm{C}=\mathrm{O}$ em éster $), 1600$ e $1580 \mathrm{~cm}^{-1}-$ $v(\mathrm{C}=\mathrm{C}$ aromático $), 1465 \mathrm{~cm}^{-1}-\mathrm{v}(\mathrm{C}-\mathrm{C}), \delta_{\mathrm{s}}\left(\mathrm{CH}_{2}\right) \mathrm{e}$ $\delta_{\mathrm{a}}\left(\mathrm{CH}_{3}\right), 1386 \mathrm{~cm}^{-1}-\delta_{\mathrm{s}}\left(\mathrm{CH}_{3}\right), 1269,1120,1072,1041$ e $1027 \mathrm{~cm}^{-1}-v(\mathrm{COOC}), 742$ e $712 \mathrm{~cm}^{-1}-\delta(\mathrm{C}-\mathrm{H}$ substituição aromática). Este conjunto de absorções confirma que a resina alquídica é o polímero-base do esmalte[13a] .

\section{Análise do pirolisado da amostra EPFA}

As principais absorções FTIR observadas no espectro do pirolisado do esmalte EPFA(Figura 2 d), após a amostra ter sido tratada com éter, estão em: 1852 e $1790 \mathrm{~cm}^{-1}-\mathrm{v}(\mathrm{C}=\mathrm{O}$ em anidrido cíclico $), 1260$, 1171,1110 e $910 \mathrm{~cm}^{-1}-\mathrm{v}$ (COOC em anidrido cíclico) e caracterizam $^{[5]}$ a presença de anidrido cíclico de 5 membros. Da mesma forma que para a amostra anterior, o espectro do pirolisado foi comparado ao do referido composto [11b] e a similaridade observada confirmou que este anidrido é um dos materiais de partida da resina.

Análise do líquido obtido após condensação de vapores da amostra EPFA

As principais absorções FTIR do líquido obtido após condensação de vapores da amostra EPFA(Figura $2 \mathrm{~g}$ ) estão em: $3027 \mathrm{~cm}^{-1}-\mathrm{v}(\mathrm{C}-\mathrm{H}), 2965 \mathrm{~cm}^{-1}-\mathrm{v}_{\mathrm{a}}\left(\mathrm{CH}_{3}\right)$, $2926 \mathrm{~cm}^{-1}-v_{\mathrm{a}}\left(\mathrm{CH}_{2}\right), 2872 \mathrm{~cm}^{-1}-\mathrm{v}_{\mathrm{a}}\left(\mathrm{CH}_{3}\right), 1606,1516$, 1495 e $1454 \mathrm{~cm}^{-1}-v(\mathrm{C}=\mathrm{C}$ aromático $), 1454 \mathrm{~cm}^{-1}$ $\delta_{\mathrm{a}}\left(\mathrm{CH}_{3}\right)$ e $\delta_{\mathrm{s}}\left(\mathrm{CH}_{2}\right), 1377 \mathrm{~cm}^{-1}-\delta_{\mathrm{s}}\left(\mathrm{CH}_{3}\right), 796,770,744$ e $697 \mathrm{~cm}^{-1}-\delta(\mathrm{C}-\mathrm{H}$ - substituição aromática $)$. Essas absorções sugerem a presença de um tipo de hidrocarboneto aromático ou de suas misturas, de estrutura similar ao encontrado na amostra ${ }^{[11 c]}$ TALP.

\section{Análise por FTIR da amostra do esmalte EPFA segundo o método 2}

As principais absorções FTIR foram observadas para o sal obtido após saponificação da resina que compõe o esmalte alquídico EPFA . Essas absorções revelam a presença de sal de ácido $\left(1603 \mathrm{e} 1572 \mathrm{~cm}^{-1}\right.$ $-\mathrm{v}_{\mathrm{a}}(\mathrm{C}=\mathrm{O}), 1406$ e $1382 \mathrm{~cm}^{-1}-\mathrm{v}_{\mathrm{s}}(\mathrm{C}=\mathrm{O})$, o que está de acordo com a metodologia (método 2 - passo 7).

As principais absorções FTIR do espectro do resíduo obtido após aplicação do método 2 (passos 10 a 16) ao esmalte estão em: $3339 \mathrm{~cm}^{-1}-\mathrm{v}(\mathrm{OH})$, $2936 \mathrm{~cm}^{-1}-v_{\mathrm{a}}\left(\mathrm{CH}_{2}\right), 2884 \mathrm{~cm}^{-1}-\mathrm{v}_{\mathrm{s}}\left(\mathrm{CH}_{2}\right), 1556 \mathrm{~cm}^{-1}$ - $\mathrm{v}_{\mathrm{a}}(\mathrm{C}=\mathrm{O}), 1464 \mathrm{~cm}^{-1}-\delta_{\mathrm{s}}\left(\mathrm{CH}_{2}\right), 1396 \mathrm{~cm}^{-1}-\delta_{\mathrm{s}}(\mathrm{C}=\mathrm{O})$, $1414 \mathrm{~cm}^{-1}-\delta(\mathrm{OH})$ e $1018 \mathrm{~cm}^{-1}-v(\mathrm{C}-\mathrm{O})$.

Este conjunto de absorções, associado ao seu formato, sugere a presença básica de composto alcoólico e ainda do sal ácido. As principais bandas observadas para o glicol são similares às do pentaeritritol, um dos poliálcoois mais utilizados ${ }^{[1,2]}$, sugerindo que este poliálcool esteja presente no esmalte EPFA (Figura $2 \mathrm{e}$ ).

As principais bandas FTIR observadas no espectro do resíduo obtido após aplicação do método 2 (passos 17 a 19) ao esmalte EPFA estão em: 3408 - 2673 $\mathrm{cm}^{-1}-\mathrm{v}(\mathrm{OH}), 2924 \mathrm{~cm}^{-1}-\mathrm{v}_{\mathrm{a}}\left(\mathrm{CH}_{2}\right), 2853 \mathrm{~cm}^{-1}-\mathrm{v}_{\mathrm{s}}\left(\mathrm{CH}_{2}\right)$, $1710 \mathrm{~cm}^{-1}-\mathrm{v}(\mathrm{C}=\mathrm{O}), 1463 \mathrm{~cm}^{-1}-\delta_{\mathrm{a}}\left(\mathrm{CH}_{3}\right)$ e $\delta_{\mathrm{s}}\left(\mathrm{CH}_{2}\right)$, $1381 \mathrm{~cm}^{-1}-\delta(\mathrm{OH})$ e $\delta_{\mathrm{s}}\left(\mathrm{CH}_{3}\right)$ e $1262 \mathrm{~cm}^{-1}-\mathrm{v}(\mathrm{C}-\mathrm{O})$. Tais bandas indicam a presença de ácido orgânico.

Essas absorções são observadas em misturas de ácidos graxos insaturados, indicando que estes compostos fazem parte da formulação do esmalte EPFA $^{[13 c]}$ (Figura 2f). 


\section{Avaliação de dados FTIR obtidos para as amos- tras TALP e EPFA}

As Figuras 1 e 2 revelam, respectivamente, as absorções dos diferentes compostos separados das amostras TALP e EPFA após aplicação da metodologia desenvolvida (métodos 1 e 2). Pode ser observado que a utilização de vários métodos de separação acoplados às diferentes técnicas FTIR permitiu a separação, e conseqüente identificação, dos principais componentes das amostras, alcançando o objetivo proposto.

Os principais componentes encontrados na tinta TALP foram: polímero base - resina alquídica contendo como materiais de partida o componente ácido, anidrido ftálico (método 1), e o glicol, pentaertinitrol (método 2); pigmento - silicato de alumínio (método 1); componente volátil - hidrocarboneto aromático (método 1) e ácido graxo - óleo de soja (método 2).

Os principais componentes encontrados no esmalte EPFA foram: polímero base - resina alquídica, contendo como materiais de partida o componente ácido, anidrido ftálico (método 1), e o glicol, pentaeritritol (método 2); pigmento - carbonato de cálcio (método 1); componente volátil - hidrocarboneto aromático (método 1) e ácido graxo - misturas de ácidos graxos insaturados (método 2).

\section{Conclusão}

O conjunto de dados avaliados mostra que a metodologia idealizada e aplicada (métodos 1 e 2) à tinta TALP e esmalte EPFA permite caracterizar a presença de materiais assumidos como referências e os principais componentes de amostras desconhecidas, dentro dos limites de detecção permitidos. Desta forma foi alcançado o objetivo proposto, sendo também demonstrada a importância do acoplamento de métodos à caracterização de tintas à base de resinas alquídicas. Entende-se portanto, que uma nova metodologia foi desenvolvida para a caracterização de amostras desconhecidas deste tipo de tintas. Mediante esses dados, é possível prever que esta metodologia possa ser também aplicada, com adaptações específicas e apropriadas, às tintas com composições similares.

\section{Referências Bibliográficas}

1. Fazenda, J. M. R. - "Tintas e Vernizes. Ciência e Tecnologia", Abrafati, São Paulo, $2^{\text {th }}$ ed 1995).

2. Othmer, K. "Encyclopedia of Chemical Technology", $3^{\text {th }}$ ed. v. 2, p.18 John Wiley \& Sons, NewYork (1978).

3. Othmer, K. - "Encyclopedia of Chemical Technology", $3^{\text {th }}$ ed., v. 6, p.427 John-Wiley, NewYork (1981).

4. Fairbanks, M.M. - Química e derivados, agosto, p. 22 (1997).

5. Smith, A.L. - "Applied Infrared Spectroscopy", JohnWiley, NewYork, (1979).

6. Anderson, D.G. - Analitycal Chemistry, v. 63, p.87R (1991).

7. Anderson, D.G. - Analitycal Chemistry, v. 65 , p.1R 11R (1993).

8. Anderson, D.G. -Analitycal Chemistry, v. 67, p.33R 46R (1995).

9. Swinehart, J.S.;. Hannah, R.W;. Perkins, W.D - "Paints and coatings". Nota Técnica Perkin Elmer Corporation, 1968.

10. Kuptsov, A.H. - Journal of Forensic Sciences, 39(2), p.305 (1994).

11. Hummel, D. O.; Scholl, F. - Atlas of Polymer and Plastics Analysis. v III, Verlag Chemie, Weinheim, (1981), a) 5405, b) 6400, c) 5296, d) 5431.

12. Othmer, K. - "Encyclopedia of Chemical Technology", $3^{\text {th }}$ ed., v. 16, p.742, John-Wiley, NewYork (1981).

13. Hummel, D. O.; Scholl, F. - Atlas of Polymer and Plastics Analysis. v II, Verlag Chemie, Weinheim, (1984), a) 2920 , b) 4159 , c) 4157 .

Recebido: $10 / 08 / 01$

Aprovado: 10/08/02 